\title{
$1 \quad$ Unsupervised deep learning method for cell segmentation
}

2

3

4

5

6

7

8 * corresponding author: jyu@uchc.edu

11 Abstract

Advances in the artificial neural network have made machine learning techniques

14 increasingly more important in image analysis tasks. Recently, convolutional neural networks

15 (CNN) have been applied to the problem of cell segmentation from microscopy images.

16 However, previous methods used a supervised training paradigm in order to create an accurate

17 segmentation model. This strategy requires a large amount of manually labeled cellular images,

18 in which accurate segmentations at pixel level were produced by human operators. Generating

19 training data is expensive and a major hindrance in the wider adoption of machine learning

20 based methods for cell segmentation. Here we present an alternative strategy that uses

21 unsupervised learning to train CNNs without any human-labeled data. We show that our method

22 is able to produce accurate segmentation models. More importantly, the algorithm is applicable

23 to both fluorescence and bright-field images, requiring no prior knowledge of signal

24 characteristics and requires no tuning of parameters. 


\section{Introduction}

Automated cellular segmentation from optical microscopy images is a critical task in many biological researches that rely on single-cell analysis. Traditional approaches to cell segmentation rely on manually-crafted feature definitions that allow the algorithmic recognition of cellular area and cell border ${ }^{1,2}$. Unfortunately manual feature definitions are usually highly context-specific and require task-dependent tuning to work well. For example, an algorithm that is well-optimized for a specific membrane fluorescent marker will not work on images of a different fluorescent marker, nor on bright field images. Switching to a different cell type (with different morphological features), changing imaging modality (e.g., from epi-fluorescence to confocal), or even imaging settings (e.g. changing the objective) often requires a redesign/reoptimization of the segmentation algorithm. Therefore, there is a need for more generic, "turn-key" solutions for this task. various kinds of image processing tasks, including semantic segmentation ${ }^{3-6}$. In particular, various networks with an hourglass-shaped architecture (e.g., UNet) have achieved remarkable pixel-level accuracy in segmentation of objects, including biological cells ${ }^{7-11}$. However, there are while CNNs perform well in segmentation of a single cell, they often exhibit an under-segmentation bias when processing crowded cell populations that contain many cells adjacent to each other ${ }^{8}$. This makes them less desirable for common analytical tasks such as high content screening, in which typically hundreds to thousands of cells need to be segmented simultaneously from one image dataset. Second, and more importantly, to train a CNN for the segmentation task, one needs a significant amount of manually labeled training images, in 49 which cell areas and/or cell boundaries are marked by human operators. Manually labeling is a 
50 time-consuming and expensive process. As a result, CNN-based approach remains

51 uncompetitive versus traditional segmentation approaches in real-life biological applications.

53 two problems described above. A key difference between our method and previous attempts at

54 the CNN-based cell segmentation is that we use a maker-controlled segmentation algorithm,

55 taking a note from conventional cell segmentation pipelines. Many conventional segmentation

56 algorithms (e.g., watershed ${ }^{12}$ ) are known to have an over-segmentation bias when the images

57 are contaminated with noise. One way to correct for the bias is to provide a set of specific

58 marker positions, depicting the approximate locations of each cell. Indeed, it is a common

59 practice to use two-channel imaging data for cell segmentation, one channel for imaging

60 nucleus and the other for the whole cell. The nucleus images are useful for computing marker

61 positions, and the whole cell images provide information for determining cell boundaries. This

62 approach is used in some of the most widely-used cell segmentation softwares, such as Cell

63 Profiler $^{13}$ and llastik ${ }^{14}$. The success of this approach derives partly from the fact that nucleus

64 images have simple morphological features and therefore are relatively easy to analyze with

65 traditional image processing algorithms. Nucleus identification and/or segmentation is a heavily

66 researched topic; multiple algorithms exist in the literature with good performances ${ }^{15-19}$. The

67 main challenge in cell segmentation is to devise reliable feature representations that can

68 recognize cell boundaries with a high accuracy.

70 CNN-based segmentation. We will first generate marker locations from nucleus images, but use

71 a CNN to model cell features and produce accurate segmentation at the whole cell level. Unlike

72 previous works on CNN segmentation, which use the networks to process the whole imaging

73 field of view, we designed our network to perform segmentation on a smaller patch of the input

74 image centered around the marker positions (Fig.1). This converts the multi-cell segmentation 
75 problem to multiple single-cell segmentation problems, which in turn removes the

76 undersegmention bias as long as the nuclei markers were correctly computed. In addition, it is

77 important to note that the "nucleus image" does not have to be one that is experimentally

78 acquired. Instead, we will also demonstrate an alternative approach, in which we generate

79 synthetic "nucleus images" from the normal whole cell images, by using a pretrained CNN

80 model. This technique is similar to the method first demonstrated by Ounkomol et. $\mathrm{al}^{20}$, in which

81 they showed that CNNs can be trained to map one image modality (e.g. bright-field image) to a

82 different one (e.g, fluorescence images of plasma membrane, nucleus etc).

A more important consequence of the marker-controlled segmentation approach is that it

84 allows us to devise a method to train segmentation CNNs (Fig. 1) in an unsupervised fashion,

85 and thus avoiding the manual labeling of the training dataset. The key for achieving this is to

86 design a custom objective function:

$87-L\left(M, y^{1}, y^{2}, \ldots, y^{n}\right)=\sum_{d_{0}, d_{1}} \sum_{i} y_{d_{0}, d_{1}}^{i}+\lambda \sum_{d_{0}, d_{1}} \sum_{i \neq j} y_{d_{0}, d_{1}}^{i} \cdot \log \left(1-y_{d_{0}, d_{1}}^{j}\right)-\beta \sum_{d_{0}, d_{1}} \sum_{i} y_{d_{0}, d_{1}}^{i}\left(1-M_{d_{0}, d_{1}}\right)$

88 Here, $y^{i} \in R^{k \times k}$ is the CNN output from $i$-th patch of the input image, which can be interpreted as

89 the probability value for classifying each pixel within the patch to be one belonging to the cell.

90 The summations over $d_{0}, d_{1}$ are over all pixels in the input image, and the summations over $i$ or $j$

91 are over all patches. Furthermore, we use the notation $y_{d_{0}, d_{1}}^{i}$ to denote the value of $y^{i}$ at a

92 specific pixel location $d_{0}, d_{1}$. Since the individual CNN output covers only a small area of the

93 entire image input, we assume a zero value when the index location $d_{0}, d_{1}$ is outside the

94 computed area, consistent with the probabilistic interpretation of $y^{i}$. Finally, $M$ is a binary mask

95 indicating the area of all cells, which can be generated from the input data with a graph cut

96 algorithm. The goal is to train CNN until the loss function $L$ is minimized. Since the loss function 
97 is designed to be fully differentiable, we can use the standard stochastic optimization ${ }^{21}$ and

98 back-propagation technique to achieve the goal in a manner similar to supervised training.

100 many pixels as possible in the segmented results. This is balanced by the second term, which

101 penalizes any overlaps of segmented pixels from different patches. Finally, the third term gives

102 additional penalties if the segmented pixels exceed the masked area, i.e, it prevents the model

103 from including the background pixels in the segmentation. The two hyper parameters, $\lambda$ and $\beta$,

104 control the relative weight of the penalty terms. Because the CNN model processes each

105 individual patch independently, when segmenting one cell, the network is unaware of the results

106 from adjacent patches. Therefore, the loss function is minimized only after the network has

107 "learned" meaningful semantic features from the images, which in turn would hopefully result in

108 correct segmentations.

\section{Results}

We implemented the proposed algorithm using the TensorFlow computational

111 framework $^{22}$ and tested its efficacy on both immunofluorescence (anti-phosphortyrosine (pY))

112 and bright-field microscopy image data. In addition, to compute marker locations, we obtained

113 nucleus images by staining cells with DNA binding dye DAPI. Traditional segmentation

114 algorithms generally do not work on bright-field images and often work poorly on noisy signals

115 such as those in immunofluorescence data. Therefore, our datasets serve as stress tests of the

116 segmentation algorithm by presenting more challenging use cases.

We train modified UNets (Fig. S1) with the acquired images to generate a segmentation

118 model. We first used the Cell Profiler software to analyze the nucleus image and computed the

119 locations of each detected nucleus as the markers, which in turn were used to produce image

120 patches (64x64 pixels), each centered around a marker location. The patch size was chosen to 
121 be larger than the size of a single cell, thus many patches have significant overlapping areas.

122 The image patches were used as the inputs to train the neural network by minimizing the loss

123 function $L$. The goal is to train the UNet to segment one single cell from each image patch.

124 However, CNN output is dependent on local information only, and is not aware of the absolution

125 positions of the pixel inputs. To ensure the segmentation apply to only one specific cell, we

126 encoded positional information in the input data by including a image of a Gaussian disk $(\sigma=$

127 15) at the marker location as an additional input channel. On top of that, in order to ensure the

128 features learned about cells are symmetric according to image orientation, we randomly flip the

129 patches along two major axises. A corresponding reverse operation was performed on the

130 network output before the computation of the loss function. For each independent dataset input,

131 we optimize a separate segmentation model based on the same underlying network architect

132 and training procedure.

134 decreases in a generally monotonic fashion until converging to a stable minimal value. The

135 overall process took between 3-6 minutes on a single computational node with a K80 GPU

136 (graphic processing unit), depending on the input. In addition, we quantified segmentation

137 accuracies by computing mean intersection over union ( $\mathrm{mIOU}$ ) between machine

138 segmentations and manual segmentations (more details below). We found that the

139 segmentation accuracy increases monotonically over the training process and reached a stable

140 maximum. Therefore, the training process did not exhibit overfitting defects, supporting the

141 validity of the loss function design for the segmentation purpose. After the loss function value

142 stabilized, we then integrated the single-patch outputs from the CNN to generate the

143 segmentation map for the whole field-of-view (Fig. 2) for both immunofluorescence input and

144 bright field input. A quick visual examination indicated that the algorithm yielded qualitatively

145 believable segmentations in both cases. 
To evaluate the segmentation quality, we performed three different quantitations of the

147

148

149

162 between two nuclei. Thus the exact information provided by the nucleus images allows quicker

163 learning of the semantic features from the cell images. Finally, for a baseline analysis, we

164 performed cell segmentation on the immunofluorescence data using Cell Profiler, a well-known

165 cell segmentation software (Table 1). Cell profiler offers several predefined algorithms for

166 unsupervised segmentation, the best of which resulted in an mIOU value of 0.69 , significantly

167 worse than our CNN model on the same fluorescence data. Cell Profiler was not designed to

168 perform segmentation on bright-field images, therefore no comparison was made for the

169 segmentations of bright-field image data. 
Next we evaluated the consistency of segmentation results on images of differential

171

172

173

174 fluorescence images or the bright-field images (Fig. 2) without the need to change parameters.

175 However, if the segmentation results were accurate, then the results should be similar, if not

176 identical, on images representing the same sample, which we found to be the case (Fig. 2e)..

177 Quantitatively, we found that the mIOU between two segmentation results, one on the anti-pY

178 immunofluorescence images and the other on bright-field images, was $0.74 \pm 0.017$. The mIOU

179 value increased to $0.809 \pm 0.051$, if the nucleus images were also included during the model

180 training. Therefore the segmentation outputs on differential image modalities indeed yielded

181 results similar to one other, further supporting the notion that the self-supervised training

182 process allows the CNN to learn correct cellular features.

183 Additionally, we tested whether models trained with one image input can perform

184 segmentation on a different image that it had not seen yet. If the unsupervised training

185 procedure allows the model to learn semantic features, then we should reasonably expect the

186 trained model to also be able to operate on a different image that is acquired in a similar

187 manner. Indeed we found that a pre-trained model can segment an unseen image producing a

188 segmentation similar to that of a "proper" model, which had previously seen the image (Fig. S3),

189 with $\mathrm{mIOU}=0.752$ for anti-pY immunofluorescence images and $\mathrm{mlOU}=0.772$ for bright-field

190 images.

Next, we explore the possibility of generating marker positions directly from the cellular

192 images themselves (i.e, not requiring a nucleus image). While the existence of the nucleus

193 image provides a convenient means to generate the marker locations, the requirement exerts

194 extra experimental overhead. We hypothesized that marker locations can also be 
195 computationally obtained, if we produce a CNN model that could produce a synthetic "nucleus"

196 image from a normal cellular image, e.g., a bright-field image of cells. We tested this idea by

197 first training a UNet to perform a mapping, either from the anti-pY immunofluorescence images

198 or from the bright-field images, to their respective nucleus images. It is important to note that our

199 goal is not to produce a realistic nucleus image, but to compute the mark locations. Thus the

200 model can be of relatively low resolution. Indeed, we found that a useful model can be obtained

201 with very little training data: i.e., two images of 1750x1750 pixels in our test. Fig. 4 shows

202 examples of synthetic nucleus images by applying pretrained models to new images the models

203 hadn't seen. While these synthetic images were far from indistinguishable from real nucleus

204 images, they reproduced the nucleus positions with enough accuracy to allow computing marker

205 locations via a simple blob detection algorithm. The marker locations were then combined with

206 the original cellular images to train a segmentation model using the unsupervised procedure

207 outlined earlier.

208 To evaluate the accuracy of the segmentation based on this synthetic marker procedure,

209 we separately quantify two sources of errors. Firstly, the model generated synthetic markers did

210 not perfectly match each individual nucleus in the sample, which resulted in either missing

211 markers (false negative) or extra markers (false positive) at wrong locations (Fig. 4). We

212 compared the synthetic marker locations with experimental images of the nuclei and found that,

213 for markers generated from immunofluorescence images, the average false negative rate is

$2141.8 \pm 0.3 \%$ and the average false positive rate os $2.2 \pm 0.3 \%$, and for markers generated from

215 bright-field images, the average false negative rate is $2.1 \pm 0.2 \%$ and the average false positive

216 rate os $2.4 \pm 0.3 \%$. These two types of errors correspond to missing cells and over-segmentation

217 of cells, respectively, in the final segmentation results. Furthermore, the segmentation model

218 had additional segmentation error at pixel level, which we evaluated by comparing the single

219 cell segmentation results with manual segmentations on selected cells. We found the mIOU 
220 value to be $0.814 \pm 0.025$ for segmenting anti-pY immunofluorescence images and $0.755 \pm 0.033$

221 for segmenting bright-field images. Interestingly, the segmentation errors produced here are not

222 significantly different from the models trained with "correct" marker locations (i.e, using

223 experimental nucleus images, see Table 1), indicating that the small amount of false-positive

224 and false-negative errors in synthetic markers had little impact on the training of the

225 segmentation models.

\section{Discussion}

227 Cellular imaging based high content screening has become more widely used in

228 biological and medical researches ${ }^{23,24}$. The ability to perform single cell segmentation accurately

229 and in a cost-effective manner is of great importance both to the commercial interests and to the

230 basic sciences of studying single cells. Currently, accurate segmentation often requires a

231 tailored algorithm specific to a fixed imaging modality, labeling procedure and specific imaging

232 settings. Deviation from an optimized protocol often resulted in lowered performances. In

233 general, these requirements significantly limit the flexibility in experimental designs. Machine

234 learning based methods, on the other hand, promise to be more flexible because they are able

235 to learn correct semantic features directly from data. Indeed, we show here that a CNN based

236 computation method can perform cell segmentation on inputs of vastly different signal

237 characteristics. In comparison to conventional algorithm-based segmentation tools, our method

238 has the advantage of having a higher accuracy and being applicable to various input signal

239 modality without the need of parameter tuning. More importantly, unlike previous CNN based

240 segmentation tools, our method does not require any human labeled "ground-truth" data for

241 training, and instead rely on an unsupervised training procedure, alleviating a significant barrier

242 in applying deep learning strategy to cell segmentation. 
The key insight from our work is that a properly designed artificial intelligence model can

244 learn semetic features from unlabeled cell image data by processing and comparing multiple

245 small image patches. The general concept of patch learning was recently introduced by several

246 highly influential studies ${ }^{25,26}$ of unsupervised machine learning. Most current works in this area,

247 however, are focusing on the spatial context between non-overlapping patches as a source of

248 free information for training the models. The training generally requires a large amount of input

249 data. On the other hand, cellular images produced in biology have significantly higher feature

250 density (i.e., they contain nothing but cells) than most natural images. We show that the unique

251 characteristics of cell images can be leveraged to reduce the training data required, to a single

252 input image in our case. Tailored to this task, our training method used overlapping image

253 patches and relied on the consistency between patches for training, resulting in a unique

254 algorithm for this task.

255 The accuracy of our method is the highest if the user also acquires nucleus images from

256 the sample, which allows computer generation of "markers" that signify the location of individual

257 cells. However, the experimental data is optional, as we show "synthetic" nucleus images can

258 be generated using a machine learning approach. The latter strategy does require pre-training

259 of a CNN, which in turn requires additional training data in the form of cellular image / nucleus

260 image pairs. We note that nominally this is a type of supervised learning, but the training

261 process does not require manually labeling of data, and uses experimentally acquired images

262 as the "ground truth" instead. The overhead involved in the training process is generally low -- in

263 our case, we showed that training with two pairs of images were sufficient to produce results

264 accurate enough for marker computation. However, the training is specific to the exact signal of

265 interest. Switching the cellular image to a different label would need retraining of the network. In

266 the end, users of the algorithm will decide which strategy (experimental vs synthetic nucleus

267 markers) is more compatible with the specific research goal at hand. 


\section{Method} squamous-cell carcinoma line A431. Cells were plated onto plasma cleaned cover glass and grown to $\sim 70 \%$ confluence in standard growth media. Cells were quickly washed once with PBS

272 and fixed with $4 \%$ paraformaldehyde for 20 min. Cells were then washed with PBS three times

273 for 5 min each, permeabilized in 1.5\% Triton-X PBS for 10 min and washed with PBS three

274 times for 10 min each. Dishes were then blocked in $1.5 \%$ BSA PBS for 60 min at $4^{\circ} \mathrm{C}$, incubated

275 with alexa647-labeled anti-pY (1:500 in blocking buffer) for $2 \mathrm{~h}$ while rocking at $4^{\circ} \mathrm{C}$, washed

276 three times for 5 min in PBS, and imaged in PBS in presence of DAPI. Epi-fluorescence and

277 brightfield images were acquired on an inverted fluorescence microscope (Olympus IX73) with

278 an 20x objective. An area of $1.4 \times 1.4 \mathrm{~mm}$ was imaged with a grid pattern for each sample,

279 resulting in stitched images of $1750 \times 1750$ pixels. The final images were stitched using the

280 stitching plugin available in the Fiji software. Datasets were collected from three sample

281 replicates.

283 locations of nuclei, using Cell Profiler (for experimental nucleus images) or using a blob

284 detection algorithm (for synthetic nucleus images). Image patches were extracted from the

285 inputs, which were either anti-pY fluorescence images or bright-field images of cells. A modified

286 UNet, used as the engine for cell segmentation, was trained to minimize the custom loss

287 function (Eq 1) with fixed hyperparameter values of $\lambda=1$ and $\beta=15$, using the adaptive

288 momentum estimation (ADAM) optimization algorithm. Typically, processing all available image

289 patches in one batch would exceed the memory capacity of a GPU, even if the patches are from

290 just one input image. Therefore, we limit each computational batch to patches within a $640 \times 640$

291 area of the input image and process the whole image in steps. Data augmentation was

292 implemented by randomly flipping and/or transposing the input patches. A corresponding 
293 reverse operation was performed on the network output before the computation of the loss

294 function. The network was trained until the loss function reaches a stable minimum. To integrate

295 individual segmentation results from patches to yield the segmentation results for the whole

296 field-of-view, each pixel was assigned to the cell with the highest probability output at that

297 location. Pixels showing segmentation probabilities less than 0.5 from all patches were assigned

298 as the background.

Binary mask. A binary mask was used during training to ensure that the segmentation of

300 individual cells will not exceed the area covered by the cells. For fluorescence images, the

301 binary mask is generated using the "Graph Cut" ${ }^{27}$ plugin in the Fiji software, which implements

302 the well-known graph-cut algorithm. The algorithm relies primarily on the gray value differences

303 to separate the foreground from the background, but compensate for the over-segmentation by

304 introducing a penalty for foreground/background boundaries. For bright-field images, the gray

305 values are not useful for distinguishing the foreground from the background. Therefore, we first

306 train a two-class RandomForest model using the "Weka Segmentation"28 Fiji plugin. We used

307 Gaussian filter, variance and laplacian values as feature inputs. We then performed a graph cut

308 on the model's probability output and used the results as the mask.

310 CNN model for producing synthetic nucleus images. The UNet models were trained with small

311 datasets of either two immunofluorescence (anti-pY) images or two brightfield images. Each

312 input image was paired with a nucleus image, which was thresholded and converted to binary

313 images. The training was stopped when the cross-entropy loss between model output and the

314 (binary) nucleus images reached the value of 0.15 . The pre-trained model was applied to new

315 input images to produce the synthetic nucleus images.

\section{Code availability}


bioRxiv preprint doi: https://doi.org/10.1101/2021.05.17.444529; this version posted May 18, 2021. The copyright holder for this preprint (which was not certified by peer review) is the author/funder. All rights reserved. No reuse allowed without permission.

318 https://github.com/jiyuuchc/cellcutter 


\section{References}

321

322

323

1. Meijering, E. Cell Segmentation: 50 Years Down the Road [Life Sciences]. IEEE Signal Processing Magazine 29, 140-145 (2012).

2. Deshmukh, B. S. \& Mankar, V. H. Segmentation of Microscopic Images: A Survey. in 2014 International Conference on Electronic Systems, Signal Processing and Computing Technologies 362-364 (2014). doi:10.1109/ICESC.2014.68.

3. Krizhevsky, A., Sutskever, I. \& Hinton, G. E. ImageNet classification with deep convolutional neural networks. Commun. ACM 60, 84-90 (2017).

4. Long, J., Shelhamer, E. \& Darrell, T. Fully convolutional networks for semantic segmentation. in 2015 IEEE Conference on Computer Vision and Pattern Recognition (CVPR) 3431-3440 (2015). doi:10.1109/CVPR.2015.7298965.

5. Ronneberger, O., Fischer, P. \& Brox, T. U-Net: Convolutional Networks for Biomedical Image Segmentation. arXiv:1505.04597 [cs] (2015).

6. Liu, Y., Minh Nguyen, D., Deligiannis, N., Ding, W. \& Munteanu, A. Hourglass-ShapeNetwork Based Semantic Segmentation for High Resolution Aerial Imagery. Remote Sensing 9, 522 (2017).

7. Al-Kofahi, Y., Zaltsman, A., Graves, R., Marshall, W. \& Rusu, M. A deep learning-based algorithm for 2-D cell segmentation in microscopy images. BMC Bioinformatics 19, 365 (2018).

8. Cameron, W. D., Bennett, A. M., Bui, C. V., Chang, H. H. \& Rocheleau, J. V. Leveraging multimodal microscopy to optimize deep learning models for cell segmentation. APL Bioengineering 5, 016101 (2021).

9. Wang, W. et al. Learn to segment single cells with deep distance estimator and deep cell detector. Computers in Biology and Medicine 108, 133-141 (2019).

10. Wollmann, T. et al. GRUU-Net: Integrated convolutional and gated recurrent neural network 
345 for cell segmentation. Medical Image Analysis 56, 68-79 (2019).

346 11. Wolny, A. et al. Accurate and versatile 3D segmentation of plant tissues at cellular

347 resolution. eLife 9, e57613 (2020).

348 12. Bertrand, G. On Topological Watersheds. J Math Imaging Vis 22, 217-230 (2005).

349 13. Carpenter, A. E. et al. CellProfiler: image analysis software for identifying and quantifying

350 cell phenotypes. Genome Biology 7, R100 (2006).

351 14. Berg, S. et al. ilastik: interactive machine learning for (bio)image analysis. Nature Methods

$35216,1226-1232$ (2019).

353 15. Wang, M. et al. Novel cell segmentation and online SVM for cell cycle phase identification in 354 automated microscopy. Bioinformatics 24, 94-101 (2008).

355 16. Sharif, J. M., Miswan, M. F., Ngadi, M. A., Salam, M. S. H. \& bin Abdul Jamil, M. M. Red

356 blood cell segmentation using masking and watershed algorithm: A preliminary study. in

3572012 International Conference on Biomedical Engineering (ICoBE) 258-262 (2012).

358 doi:10.1109/ICoBE.2012.6179016.

359 17. Ulman, V. et al. An objective comparison of cell-tracking algorithms. Nature Methods 14, $360 \quad 1141-1152(2017)$.

361 18. Dzyubachyk, O., Niessen, W. \& Meijering, E. Advanced level-set based multiple-cell 362 segmentation and tracking in time-lapse fluorescence microscopy images. in 2008 5th IEEE 363 International Symposium on Biomedical Imaging: From Nano to Macro 185-188 (2008). 364 doi:10.1109/ISBI.2008.4540963.

365 19. Al-Kofahi, Y., Lassoued, W., Lee, W. \& Roysam, B. Improved Automatic Detection and 366 Segmentation of Cell Nuclei in Histopathology Images. IEEE Transactions on Biomedical 367 Engineering 57, 841-852 (2010).

368 20. Ounkomol, C., Seshamani, S., Maleckar, M. M., Collman, F. \& Johnson, G. R. Label-free 369 prediction of three-dimensional fluorescence images from transmitted-light microscopy.

$370 \quad$ Nature Methods 15, 917-920 (2018). 
371 21. Kingma, D. P. \& Ba, J. Adam: A Method for Stochastic Optimization. arXiv:1412.6980 [cs]

372 (2017).

373 22. Martín Abadi et al. TensorFlow: Large-Scale Machine Learning on Heterogeneous Systems.

374 (2015).

375 23. Boutros, M., Heigwer, F. \& Laufer, C. Microscopy-Based High-Content Screening. Cell 163, 376 1314-1325 (2015).

377 24. Zanella, F., Lorens, J. B. \& Link, W. High content screening: seeing is believing. Trends in 378 Biotechnology 28, 237-245 (2010).

379 25. Doersch, C., Gupta, A. \& Efros, A. A. Unsupervised Visual Representation Learning by 380 Context Prediction. in 1422-1430 (2015).

381 26. Noroozi, M. \& Favaro, P. Unsupervised Learning of Visual Representations by Solving 382 Jigsaw Puzzles. in Computer Vision - ECCV 2016 (eds. Leibe, B., Matas, J., Sebe, N. \& 383 Welling, M.) 69-84 (Springer International Publishing, 2016).

384 doi:10.1007/978-3-319-46466-4_5.

385 27. Boykov, Y. \& Kolmogorov, V. An experimental comparison of min-cut/max- flow algorithms 386 for energy minimization in vision. IEEE Transactions on Pattern Analysis and Machine 387 Intelligence 26, 1124-1137 (2004).

388 28. Arganda-Carreras, I. et al. Trainable Weka Segmentation: a machine learning tool for 389 microscopy pixel classification. Bioinformatics 33, 2424-2426 (2017). 


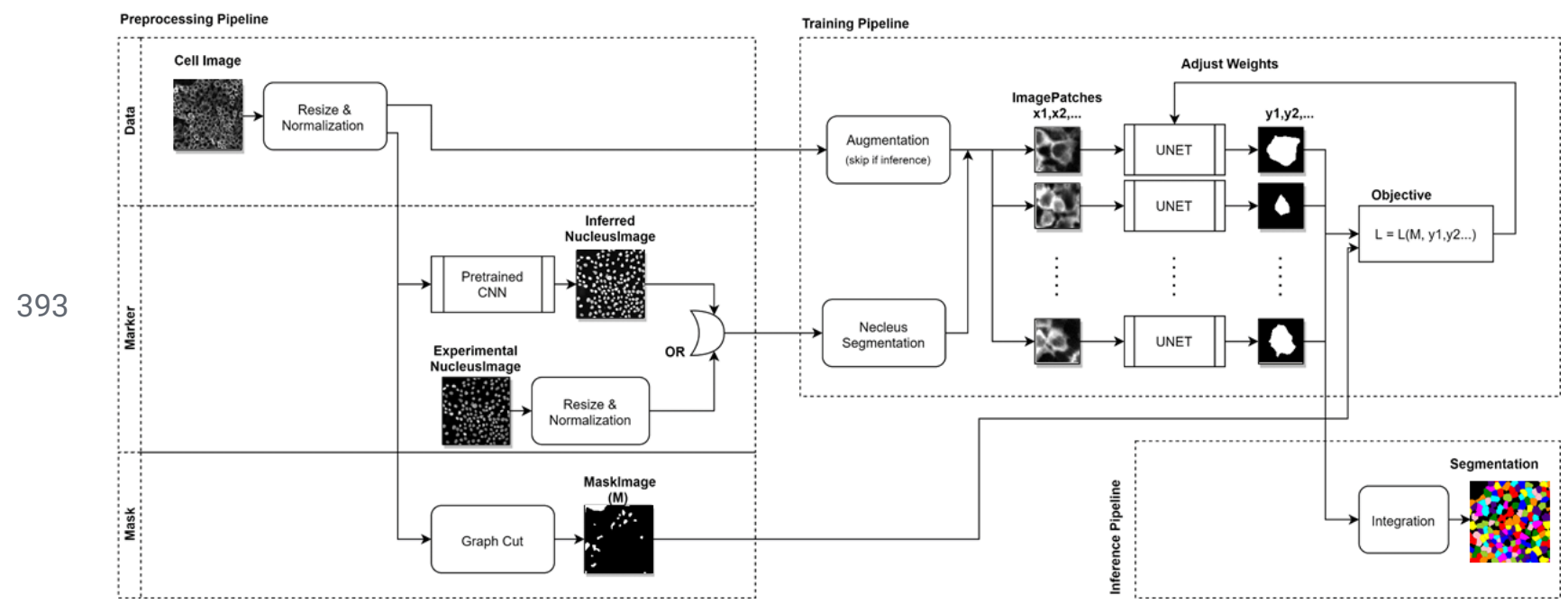

394 Figure 1. Schematic outline of the CNN segmentation algorithm. The overall process comprises

395 three components: the preprocessing pipeline, which computes the marker location and image

396 mask from the inputs, the training pipeline which extract image patches to train a CNN model for

397 segmentation, and the integration pipeline that takes the single patch output from the CNN and

398 create whole-field segmentation results. 
a

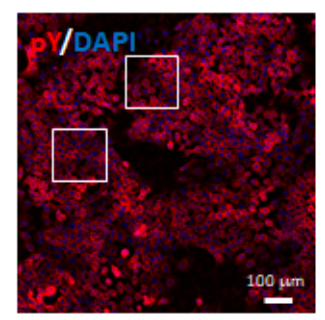

C

400
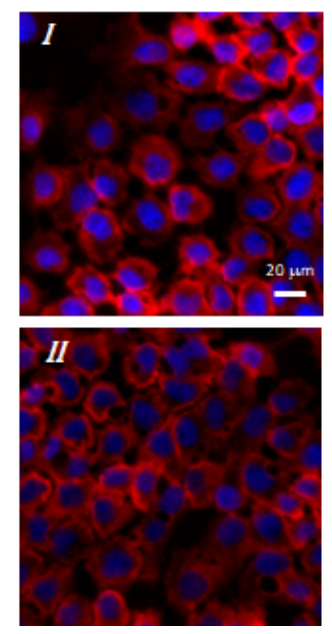

b
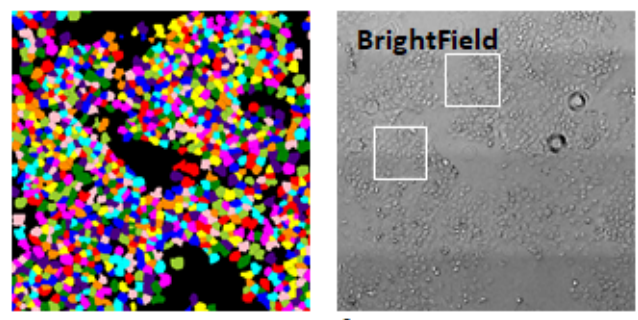

d
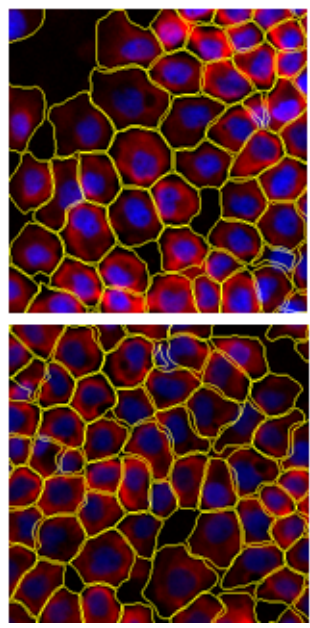

d

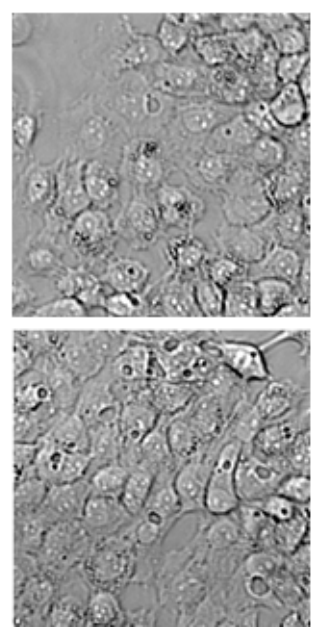

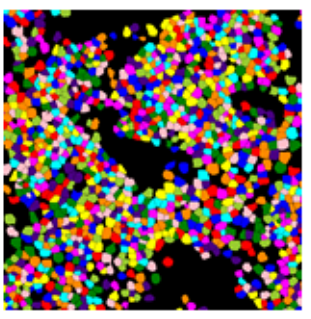

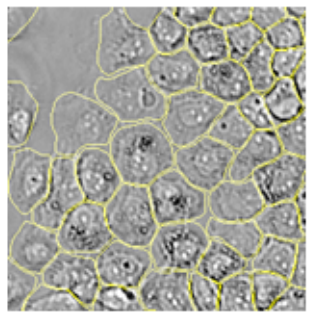

e

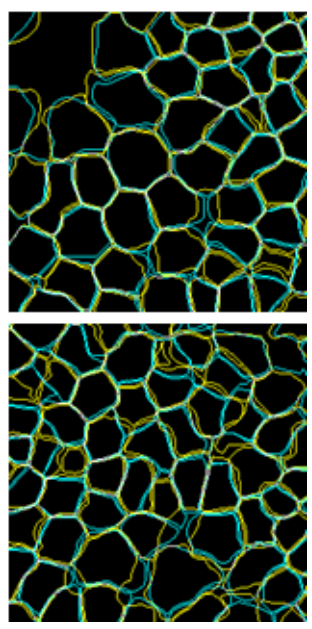

401 Figure 2. Segmentation of microscopy images by the CNN. Anti-pY immunofluorescence (a)

402 and bright-field (b) images of A431 cells were acquired from the same sample location and the

403 CNN segmentation results are shown as color maps (a and b, right panels). (c \& $\mathbf{d})$ Zoomed-in

404 view of the segmentation results from the two subregions denoted by the white boxes in $\mathbf{a} \& \mathbf{b}$.

405 Here only the outline of the segmentations were plotted as image overlays (c \& d, right

406 columns). (e) Comparisons of the segmentation results based on the immunofluorescence

407 images (yellow) versus the bright-field images (cyan). Data depict the same sub-regions as

408 shown in c \& d. All CNN segmentation models were trained with either the immunofluorescence

409 image or the bright-field image only. DAPI images (shown in blue in a \& c) were used for

410 determining marker locations, but not used in training the CNN model. 


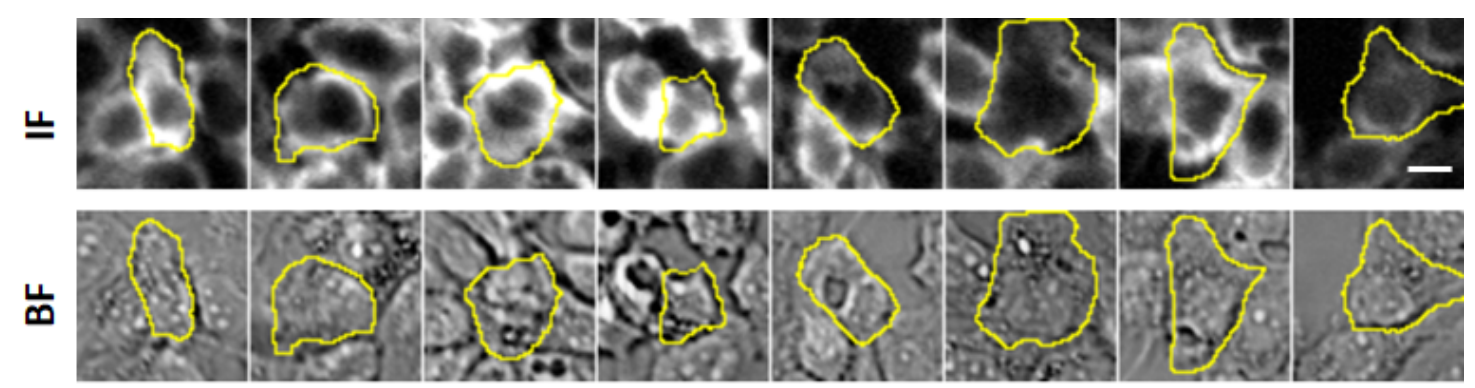

412
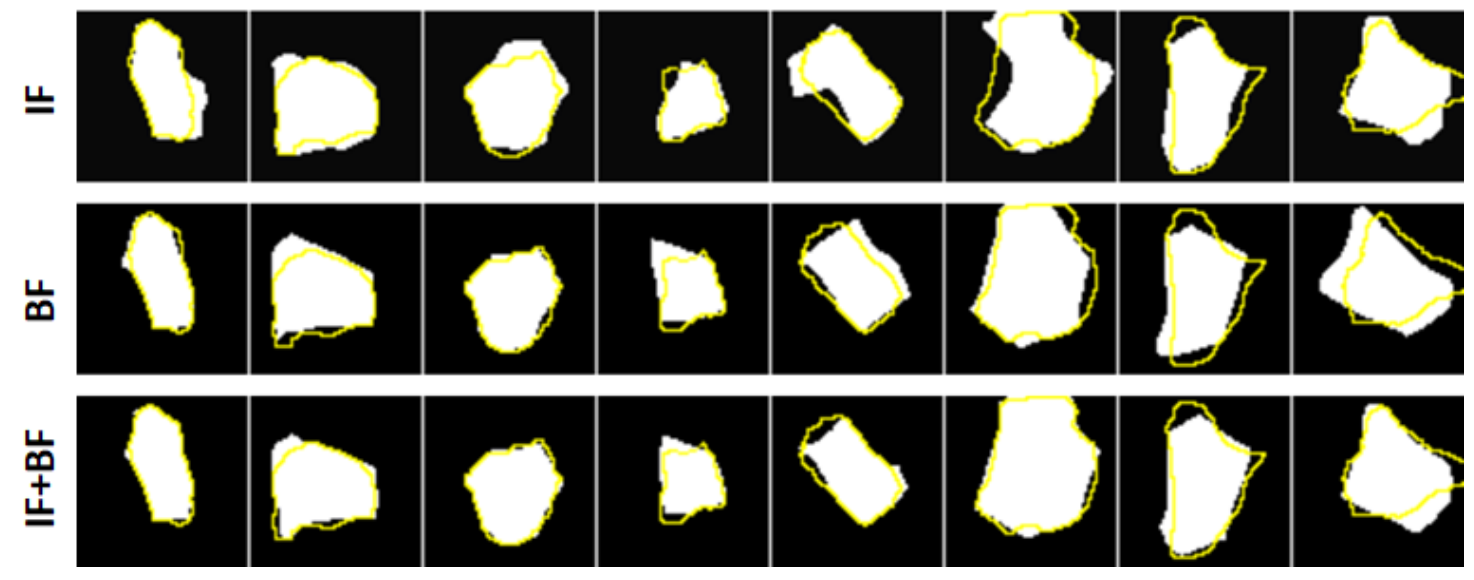

413 Figure 3. Comparisons between machine and manual segmentation results. Top two rows show

414 the examples of individual image patches from the training data. The first row shows the

415 patches from the anti-pY immunofluorescence (IF) images. The second row shows the patches

416 from the bright-field (BF) images. The bottom three rows show the CNN segmentation output of

417 each image patch. The models were trained with either the IF (row 3), BF (row 4) or

418 multi-channel input of IF and BF combined (row 4). In all cases, the manual segmentations of

419 the cells were plotted as the yellow overlays. Manual segmentation is based on a combined

420 input of IF, BF and nucleus (DAPI) images (not shown here). The scale bar represents $20 \mu \mathrm{m}$. 

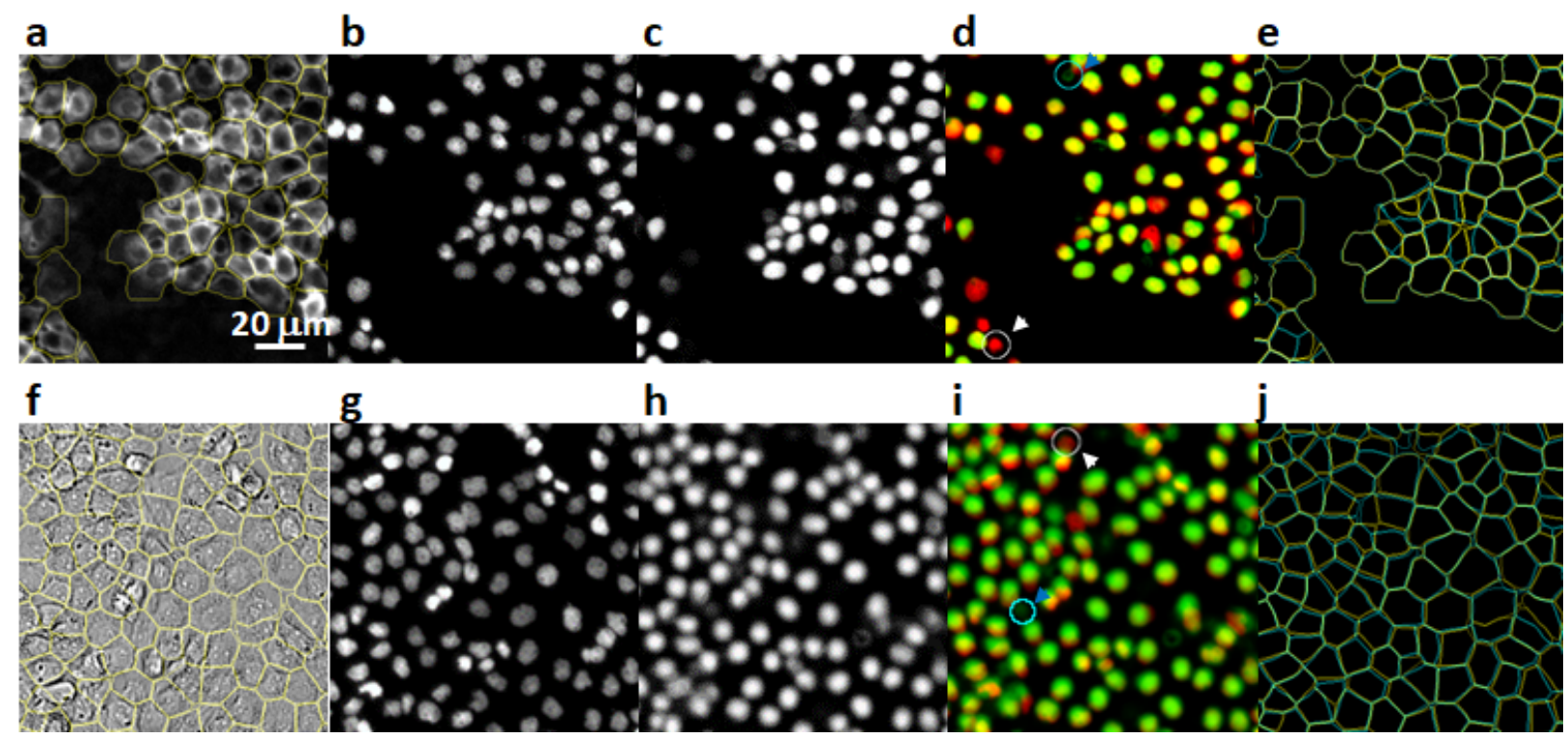

423 Figure 4. Segmentation based on synthetic marker locations. Results for the anti-pY

424 immunofluorescence data are shown in panel a-e, and results for the bright-field image are

425 shown in(f-j). The input images (a \& f) were shown together with the segmentation results

426 (yellow overlay). Experimentally acquired nucleus (DAPI) images were shown in $\mathbf{b}$ \& $\mathbf{g}$ for

427 comparison, although they were not used for the computation of the segmentation. Instead,

428 synthetic nucleus images (c \& h) were computed directly from the input images (a \& f) using a

429 pre-trained CNN. Errors in the synthetic images can be easier discerned in the composite

430 overlay images (d \& i) of the experimental (red) and synthetic (green) nucleus images. Both

431 false negative (white arrow) and false positive (blue arrow) errors were found, but are of

432 relatively low occurrences. Finally, the segmentation results based on synthetic markers were

433 compared with the "proper" segmentation, for which the experimental DAPI images were used

434 for marker locations. The comparisons were shown in e \& j, where segmentations based on

435 experimental markers (yellow) were drawn in overlay on segmentations based on synthetic

436 markers (cyan). 
bioRxiv preprint doi: https://doi.org/10.1101/2021.05.17.444529; this version posted May 18, 2021. The copyright holder for this preprint (which was not certified by peer review) is the author/funder. All rights reserved. No reuse allowed without permission.

438

439 Table I. Unsupervised CNN segmentation accuracy.

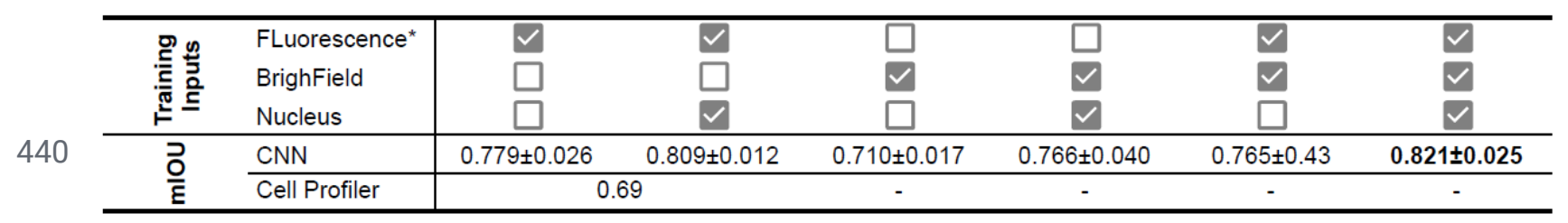

* anti-pY immunofluroescence images. 


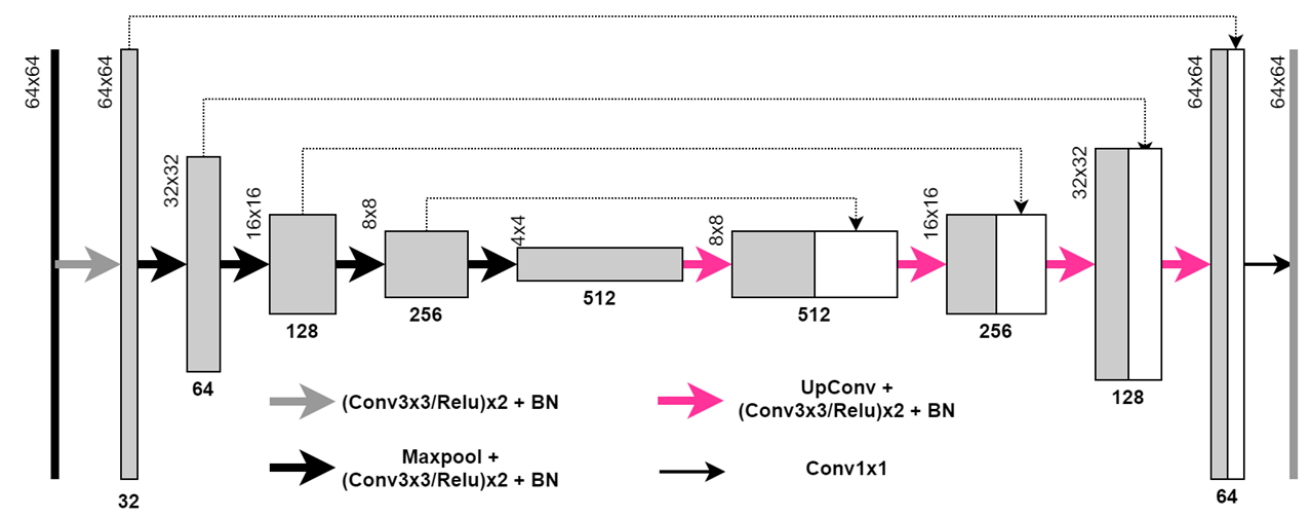

445 Figure S1. Architecture of the CNN used for segmentation. The encoder portion of the network

446 extracts image features by multiple stages of convolution and max pooling. The decoder portion

447 of the network constructs the segmentation by incremental upscaling of the feature map (to $2 x$

448 larger dimension) followed by convolution. Feature map obtained in the decoding step is

449 concatenated by intermediate results at the decoding steps in order to supplement feature

450 information at incrementally higher resolution. Relu activation is used throughout the encoding

451 and decoding steps, except in the final output layer, in which a sigmoid activation is used. The

452 same network is used for producing synthetic nucleus images, in which case the input tensor

453 dimension is much larger $(870 \times 870)$ to match the size of training data. 

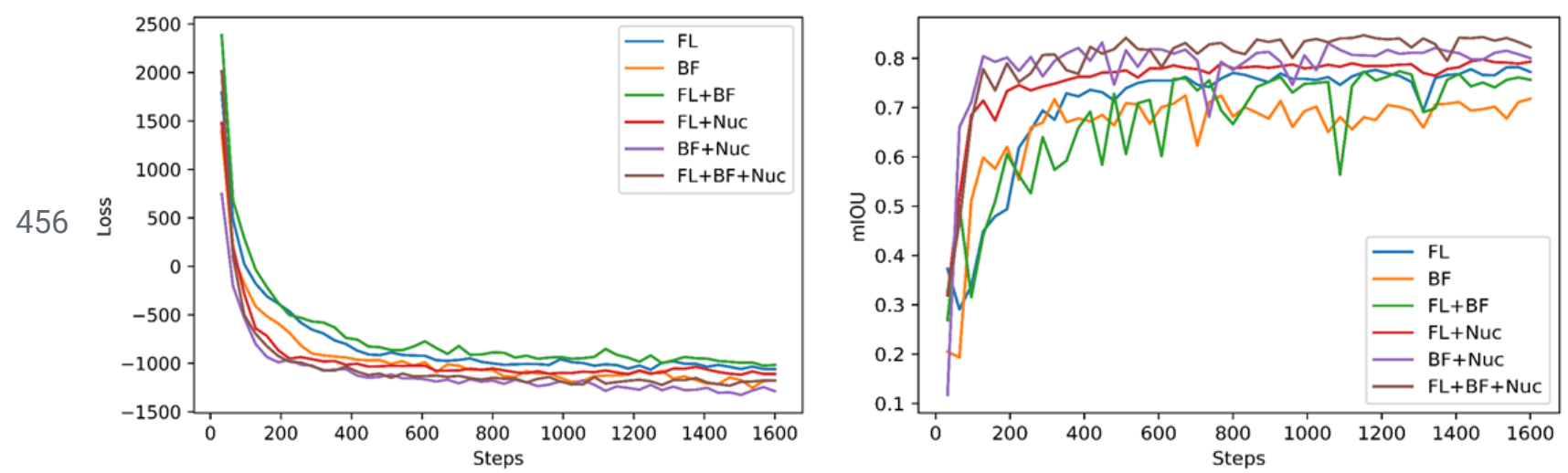

457 Figure S2. Representative training traces showing the minimization of the loss function (left)

458 and the corresponding optimization of the segmentation accuracies (right) over the

459 self-supervised training process. Multiple models were trained with various combinations of

460 input data, including the immunofluorescence image (FL), the bright-field image (BF) and the

461 DAPI staining image (Nuc). 


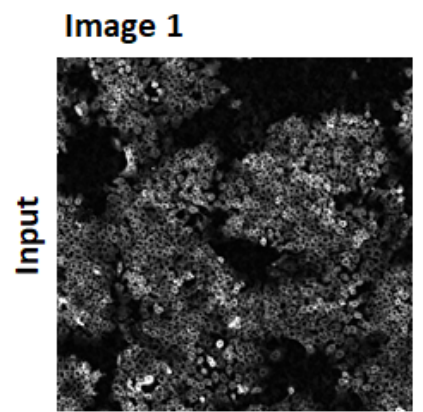

$$
\text { Image } 2
$$

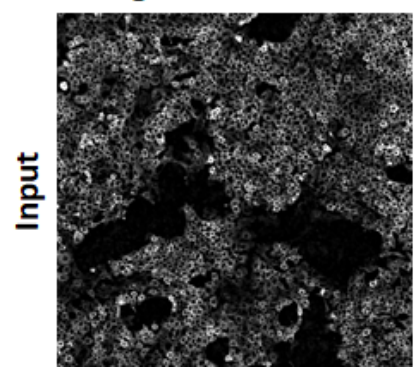

Trained with Image 1

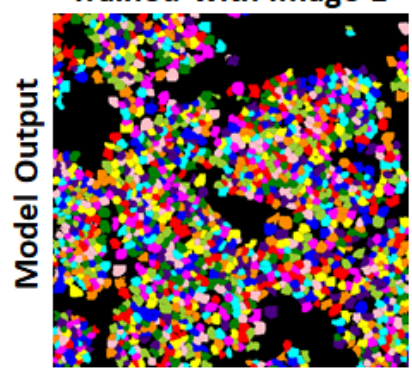

Trained with Image 1

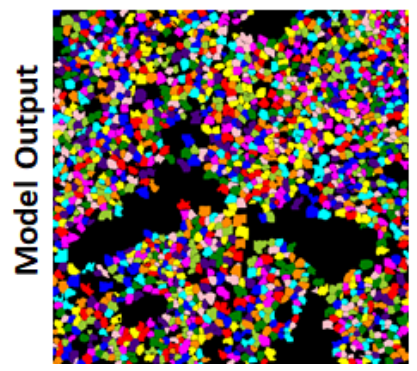

Trained with Image 2

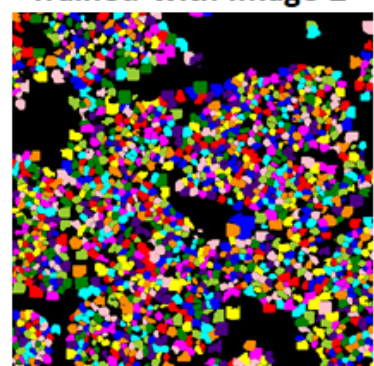

Trained with Image 2

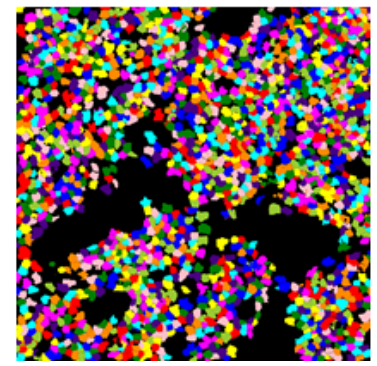

466 Figure S3. Comparison of segmentation results on both seen and unseen fluorescence images.

467 Two segmentation models were separately trained by two immunofluorescence images of the

468 same modality (left) and the resulting models were used to operate on both images (one seen

469 and one unseen) to obtain the segmentation (right). The results demonstrated that the models

470 trained this way were able to apply learnings to unseen data of similar features. 


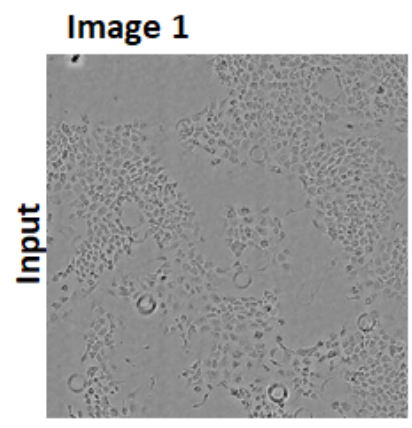

Image 2

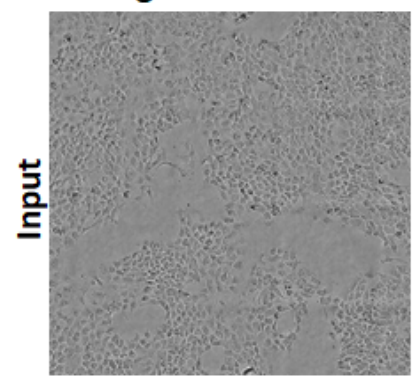

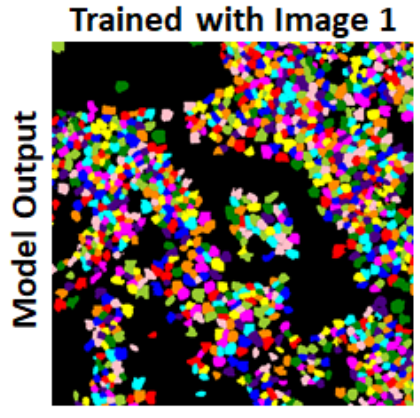

Trained with Image 1

Trained with Image 1

Trained with Image 2

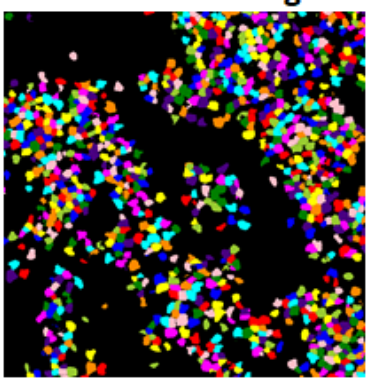

Trained with Image 2
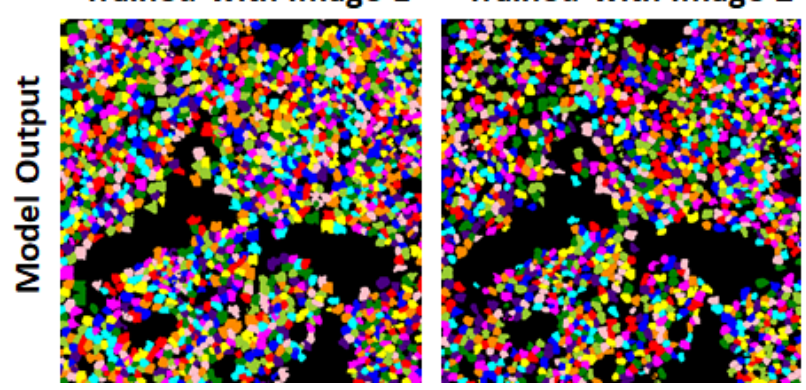

473 Figure S4. Comparison of segmentation results on both seen and unseen bright-field images.

474 This is the same analysis as in Fig S3 except on bright-field images. 